\title{
No Superior Movement of Thyroid Cartilage During Swallowing
}

National Cancer Institute

\section{Source}

National Cancer Institute. No Superior Movement of Thyroid Cartilage During

Swallowing. NCI Thesaurus. Code C127235.

A finding of no superior movement of thyroid cartilage during swallowing. 